\title{
Accident Prevention, Detection and Reporting $\cong$ for Two Wheeler Safety System
}

\author{
Kalyanaraman B, Shivam Choudhary, Malkeet Singh, Kumar Divyank
}

\begin{abstract}
This paper manages the improvement of a security framework which is coordinated with the keen protective cap and shrewd bicycle to lessen mishaps and other alcoholic and drive cases for bikes. Here weight sensor and the accelerometer sensor checks whether the individual wears the head protector or not. Additionally, at the equivalent, a liquor sensor is utilized to distinguish the alcoholic substance from the rider's breath. The vehicle won't start if the rider isn't wearing a protective cap or if the rider is plastered. At whatever point the rider crashes, the head protector hits the ground and the sensor recognizes the movement and reports the mishap case data to the relatives of the rider through IoT.
\end{abstract}

\section{INTRODUCTION}

Starting with the statement "BE CAUTIONS EVERY LIFE IS PRECIOUS" the principle explanation behind the street mishaps is a result of our recklessness and over speed. So as to survive such a circumstance here we are utilizing a few sensors, for example, pressure sensor, accelerometer sensor, what's more, liquor sensors to check whether the rider wears head protector or on the off chance that he is smashed or not and assuming so the vehicle won't get start. In this manner, we can lessen mishaps and thus our goal is to create a bike wellbeing framework that focuses on mishap This research establishes the preliminary circulate towards a fashionable part paintings of the programmed research of data accrued in the course of anticipation, recognition and revealing and to lessen the likelihood of motorbike mishaps and the chance of alcoholic and drive instances. Naturalistic riding examinations (NDS). While the goal of on the net fall area is the early growth of a far flung air-\% a coat or any prepared framework, the motivation behind disconnected event vicinity and acknowledgment are absolutely exceptional.

It intends to provide road security professionals with desire assist apparatuses that permit them to evidently technique the NDS information and to separate express driving occasions, as an instance, fundamental ones. Something else, analysts have to audit the video movie recorded during an NRS test to physically understand the beginning and stop instances of each basic condition skilled through a rider all through his/her

Revised Manuscript Received on May 15, 2020.

* Correspondence Author

Kalyanaraman $\mathbf{B}^{*}$, Assistant professor of Information technology in SRM IST,Chennai,Tamil Nadu,India. Email: kalyanab1@srmist.edu.in.

Shivam Choudhary, department of Information Technology,SRM IST,Chennai,Tamil Nadu,India. Email: shivam.dav.choudhary@gmail.com

Malkeet Singh, department of Information Technology,SRM IST,Chennai,Tamil Nadu,India. Email: malkeetsingh0130@gmail.com

Kumar Divyank, department of Information Technology,SRM IST,Chennai,Tamil Nadu,India.Email: kdivyank9@gmail.com

(C) The Authors. Published by Blue Eyes Intelligence Engineering and Sciences Publication (BEIESP). This is an open access article under the CC BY-NC-ND license (http://creativecommons.org/licenses/by-nc-nd/4.0/) moves and afterward examine the riders' self-revealed practices with their real practices. This extraction venture is tedious and exacting. The goals and tactics are unique to the extent that here we don't endeavour to apprehend occasions, as an instance, riding in a instantly line, on a curve or an indirect but as an alternative activities that appear whilst riding a PTW, recognizing standard driving and simple activities, for example, falls or near falls.

The important commitments of this paper are as in keeping with the following:

- The disconnected programmed discovery of fundamental driving activities is formalized as an difficulty of spotting changes within the suggest and the fluctuation of the symptoms created by way of the speeding up and rakish pace sensors.

We advocate a two-prepare method:

1) the programmed division of signs and symptoms to distinguish a few time companies that relate to homogeneous running structures

2) An association stage to employ every fragment to one of the instructions (usual

riding, fall or close to fall).

- An on line fall identification technique, for which unique consideration turned into paid to the effortlessness, power and registering time, with the possibility of continuous usage is created. The proposed procedure makes use of a exquisite method normally applied for consecutive discovery, particularly, manage outlines and, all the extra particularly, the multivariate mixed aggregate.

The the rest of this paper is taken care of out as follows: inside the II phase, we can display a portrayal of the prevailing state of PTW-associated research. The IV section subtleties the approach technologies that are proposed for tackling the troubles of the disconnected location/order of primary activities and on the web vicinity of falls. The $\mathrm{V}$ segment will give an research moreover, an outline of the sizeable number of consequences were given from true statistics. The VI section wraps the paper up and portrays a few achievable outcomes for future studies.

\section{Associated}

\section{Works}

Programmed vehicle checking has ended up being a critical scene in the present years. It might form into a chance by executing the accompanying innovations. This task focuses to propose a framework, which recognizes speeding vehicles over a particular speed confine and quickly reports to concerned specialists. At present, street mishaps rates have raised along these lines, there is a need for building up a framework that distinguishes an over speeding vehicle.

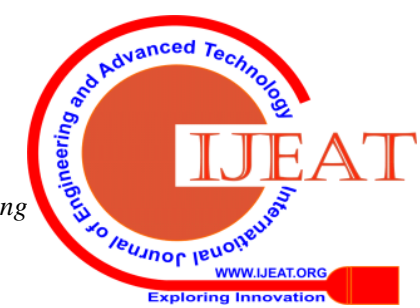


Thousands lose their significant life in vehicle mishaps consistently because of the traffic, street condition and speed. By utilizing GPS receiving wire and the most recent mechanical idea of IoT, a mishap can be quickly revealed, decreased and human life can be spared; by interfacing various types of sensors to various parts or places of a vehicle. In-tern by making these sensors speak with the emergency clinic or rescue vehicle prompt treatment can be given to the harmed individual during a mishap.

As of late, examples of mishaps, particularly on street mishaps have been extremely normal, with reports showing the consider to be high as 17 passing's for each hour on an average(in India). A few of such mishaps cause passing's because the term between the event of a mishap and informing the concerned authority is extensively bigger than what ought to be prompt. As per considers performed on an informational collection from Missouri, US [1], [2] it is expressed that the police deferred 5 mins or more to start the salvage activity in 10 to $20 \%$ of deadly mishaps.

The expansion in the populace prompts an increase in contamination and mishap too. Mechanical advancement puts forth extraordinary attempts to recognize the mishap and screen the expanding contamination. This paper depends on an upgrade in the brilliant vehicle framework. Various units actualized right now improve the vehicular framework. The principle objective is to distinguish mishaps continuously and limit the reaction time of clinical assistance. For mishap shirking, tire pressure is estimated while mishap recognition is actualized with the assistance of hub MCU.MQ7 is utilized to screen the contamination.

These days, the quantity of mishaps has expanded quickly. Around 17 mishaps happen each hour. Bicycle mishaps establish a significant lump all things considered; this is because bikes don't have the same number of wellbeing parameters which are remembered for four-wheelers. Reasons causing it very well maybe because of not wearing a cap, feeling sleepy while driving, liquor utilization, two vehicles coming into nearer closeness without the two drivers' notification, breaking of traffic signals, driving without a substantial or no driving permit, indiscreet driving, unintended activating of the increasing speed pedal, and so on.

\section{EXISTING SYSTEM}

In the current framework, it points in the mishap recognition and warning. Identification is made by utilizing a few sensors and electronic administrations. The vehicle area is acquired by utilizing GPS. Wearing head protector or not and there is no endeavour to forestall the alcoholic and drive cases.

Earlier, there has been a very little research on accident detection and prevention countermeasures. The framework that was created was unable in providing with correct results to enhance the safety of two-wheeled riders. Technology could also be called as an issue here. Some of the solutions were made but it could not suit majority of the riders. There were many different variations of tests being performed after creating certain simulations and calculations but nothing contribute much.

Disadvantage:

- Less exactness in the recognition of a mishap.

- There is no framework to check if the rider is wearing the protective cap or not.
- There is no endeavour to forestall alcoholic and drive cases.

\section{PROPOSED SYSTEM}

Right now, the two sensors for checking if the rider wears a protective cap and to check on the off chance that he is intoxicated or not. It for the most part points in the counteraction, discovery, and revealing of mishaps. Here we are utilizing nodemcu. On the off chance that a mishap is identified alarm message will be sent to the concerned individual utilizing MQTT correspondence. This framework, for the most part, incorporates 3 units cap unit, bicycle unit, recipient unit. Some of the advantages are mechanized framework, can get a caution from any remote area, GPS based finding, minimal effort usage.

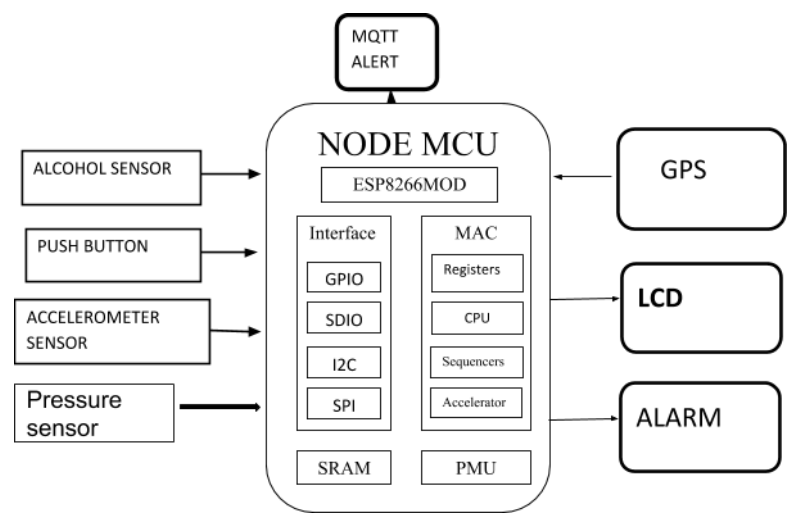

Fig.1- Block diagram of NODE MCU

\section{A. .ESP8266MOD}

\section{- Where to use ESP8266-01}

The ESP8266 is very easy to use and minimal effort gadget to give web network to your ventures. The module can work both as an Access point (can make a hotspot) and as a station (can associate with $\mathrm{Wi}-\mathrm{Fi}$ ), subsequently it can without much of a stretch bring information and transfer it to the web making the Internet of Things as simple as would be prudent. It can likewise get information from the web utilizing API's subsequently your task could get to any data that is accessible on the web, in this way making it more astute. Another energizing component of this module is that it tends to be customized utilizing the Arduino IDE which makes It extensively more clean to apply. Anyway, this form of the module has just 2 GPIO pins (you may hack it to utilize some thing like 4) so you need to utilize it alongside every other microcontroller like Arduino, else you can check out the more independent ESP-12 or ESP-32 variants. So on the off risk which you are attempting to find a module, first of all IoT or to give net network to your undertaking then this module is the right choice for you.

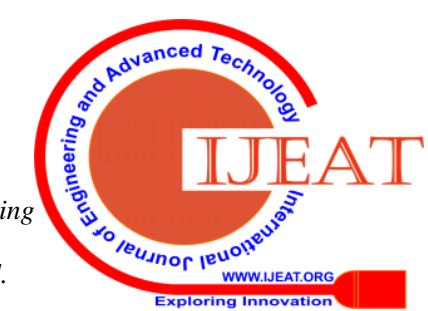


How to apply ESP8266 module

There are so many techniques and IDEs available to with ESP modules, but the most commonly used on is the Arduino IDE. So allow us to speak simplest approximately that in addition under.

The ESP8266 module works with three.3V best, something greater than $3.7 \mathrm{~V}$ might kill the module for this reason be cautions with your circuits. The pleasant manner to software an ESP-01 is by the usage of the FTDI board that helps 3.3V programming. If you don't have one it's miles advocated to buy one or for time being you could also use an Arduino board. One usually problem that each one faces with ESP-01 is the powering up hassle. The module is a piece power hungry while programming and hence you can power it with a three.3V pin on Arduino or just use a capacity divider. So it is vital to make a small voltage regulator for $3.31 \mathrm{v}$ that might supply not less than $500 \mathrm{~mA}$. One advocated regulator is the LM317 which could take care of the activity easily. A simplified circuit diagram for the use of the ESP8266-01 module is given below

- The transfer SW2 (Programming Switch) need to be held pressed to preserve the GPIO-zero pin to floor. This way we can enter into the programming mode and upload the code. Once the code is

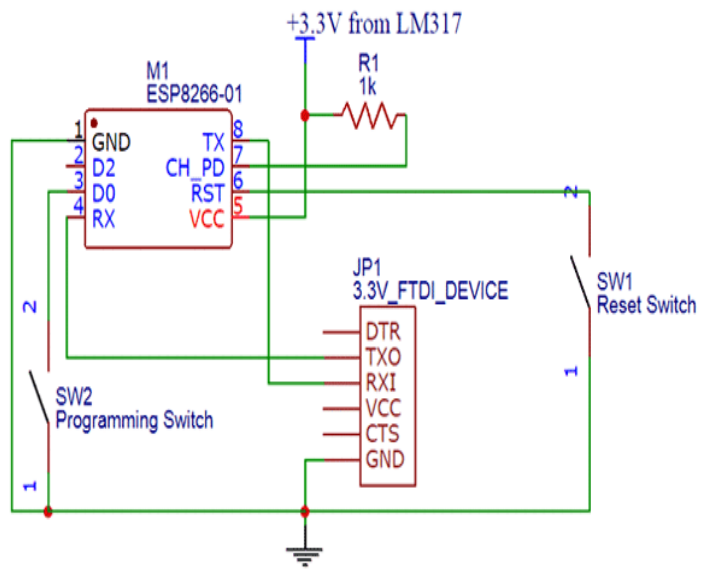

Fig.2-Circuit diagram of ESP8266-01

launched the transfer can be released.Power Requirements of ESP8266

As the operating voltage range of ESP8266 is $3 \mathrm{~V}$ to $3.6 \mathrm{~V}$, the board comes with a LDO voltage regulator to keep the voltage steady at $3.3 \mathrm{~V}$. It can reliably supply up to $600 \mathrm{~mA}$, which should be more than enough when ESP8266 pulls as much as $80 \mathrm{~mA}$ during RF transmissions. The output of the regulator is also broken out to one of the sides of the board and labelled as 3V3. This pin can be used to supply power to external components.

- $\quad$ Operating Voltage: $2.5 \mathrm{~V}$ to $3.6 \mathrm{~V}$

- On-board 3.3V 600mA regulator

- $\quad$ 80mA Operating Current

- $20 \mu \mathrm{A} n$ the course of Sleep Mode

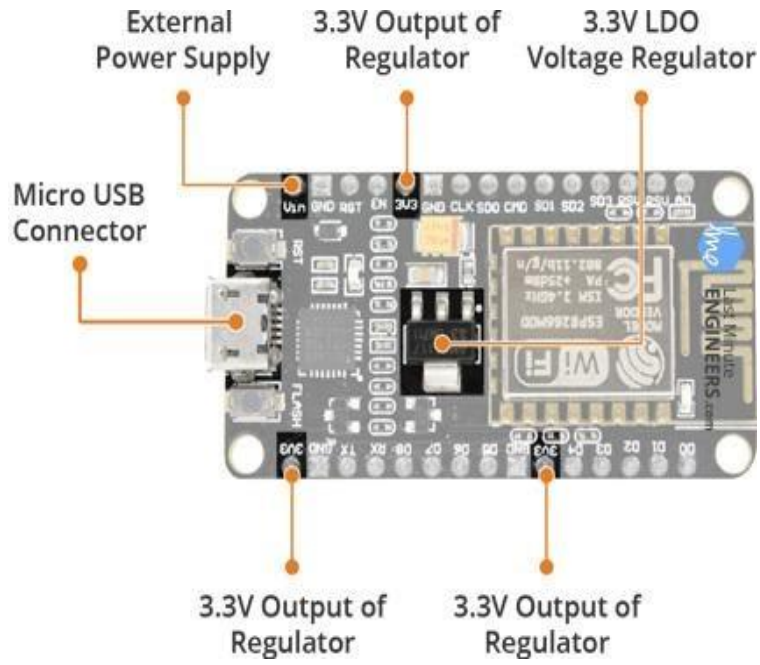

Fig.3-Energy board

Power to the ESP8266 NodeMCU is furnished thru the onboard MicroB USB connector. Alternatively, if you have a regulated $5 \mathrm{~V}$ voltage source, the VIN pin can be used to directly supply the ESP8266 and its peripherals.

\section{B..MQTT (Message Queuing Telemetry Transport)}

\section{- Introduction}

MQTT decouples the publisher from the subscriber, patron connections are continually treated via a dealer. Before we get into the info of those connections, permit's be clean what client and dealer method.

Client- Anyone who is a person of MQTT offerings are the customers. Both writer and subscriber are the customers. It simply helps in differentiating who's at the publishing give up and who's the subscriber. An MQTT purchaser is any device (from a micro controller as much as a fullfledged server) that runs an MQTT library and connects to an MQTT dealer over a network. MQTT client libraries are well capable of strolling many programming languages as, Android, Arduino, C, C++, C\#, Go, iOS, Java, JavaScript, and .NET.

Broker-It's the counterpart of the MQTT purchaser. The broker is centres of any put up/subscribe protocols. A broking can handle up to heaps of concurrent protocols, depending on the way it's been carried out. The broker is responsible for receiving all messages, filtering the messages, determining who is subscribed to every message, and sending the message to these subscribed clients.

\section{- . MQTT Connections}

The MQTT protocol is based on TCP/IP. Both the customer and the broker need to have a TCP/IP stack. The MQTT connection is usually between one consumer and the dealer. Clients by no means hook up with every other directly. To provoke a connection, the patron sends a CONNECT message to the dealer.

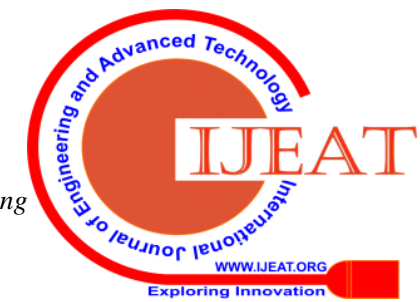




\section{Accident Prevention, Detection and Reporting For Two Wheeler Safety System}

The dealer responds with a CONNACK message and a status code. Once the connection is established, the dealer keeps it open till the customer sends a disconnect command or the relationship breaks.

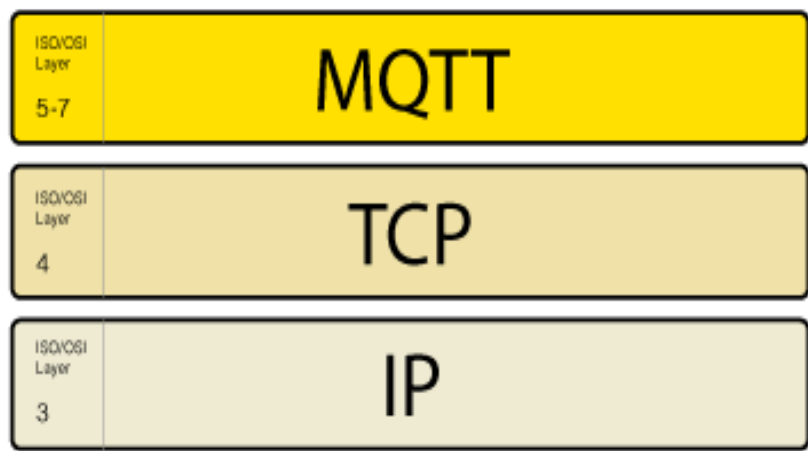

Fig.4-Basic MQTT connection protocol

- MQTT Protocols and use cases

Facebook currently makes use of MQTT for its Messenger app, not best due to the fact the protocol conserves battery electricity in the course Of cellular mobile phone-tocellphone messaging, however also because the protocol permits messages to be delivered correctly in milliseconds (ms), in spite of inconsistent internet connections throughout the globe. Most predominant cloud services companies, including Amazon Web Services (AWS), Google Cloud, IBM Cloud and Microsoft Azure, assist MQTT. It is nicely appropriate to packages the use of M2M and IoT devices for purposes which includes actual-time analytics, preventative renovation and monitoring in environments, inclusive of smart houses, healthcare, logistics, enterprise and production.

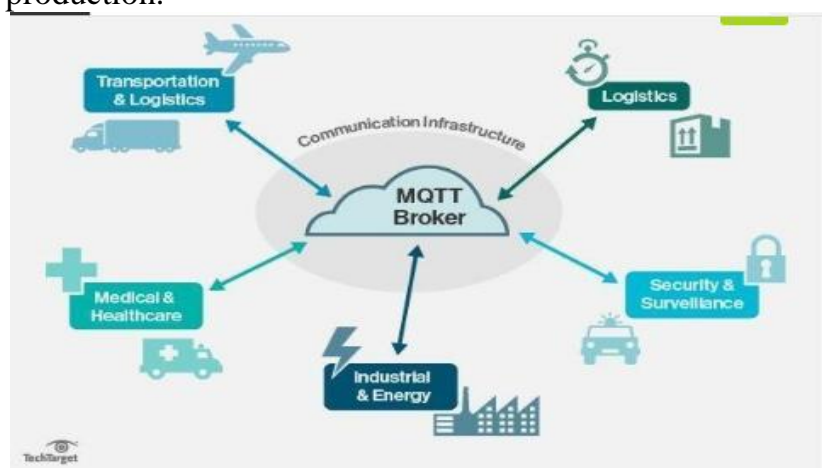

Fig.5-Publisher/Subscriber communication infrastructure

- MQTT in IoT

MQTT is one of the most usually used protocols concerning IoT. MQTT allows resource-constrained IoT gadgets to ship, or put up, records approximately a given subject matter to a server that capabilities as an MQTT message broking. The dealer then pushes the records out to those customers which have previously subscribed to the topic. To a human, a subject looks like a hierarchical record route. Clients can join a particular stage of a topic's hierarchy or use a wildcard person to subscribe to more than one degrees.

Other transfer protocols that compete with MQTT include the following:
- $\quad$ Constrained Application Protocol (CoAP)

- $\quad$ Advanced Message Queuing Protocol (AMQP)

- Simple/Streaming Text Oriented Messaging Protocol (STOMP)

- Mosquitto

- $\quad$ Simple Media Control Protocol (SMCP)

- $\quad$ SSI (Simple Sensor Interface)

- $\quad$ Data Distribution Service (DDS)

\section{RESULT AND DISCUSSION}

Our system is more enhanced and accurate than the previous systems. It can detect the motion of the bike or the wearing of helmet by the rider more precisely. Our system also detects that if the rider had alcohol, to reduce the risk of accident due to drink and drive cases.

\section{CONCLUSION}

The bike security framework created with a brilliant protective cap and astute biking framework is solid and intends to help in the counteraction, recognition, and revealing of mishaps additionally decreasing the likelihood of the alcoholic and drive cases. It likewise has a few preferences contrasted with the past frameworks. Our proposed framework gives the essential significance of forestalling mishaps and guarantees wellbeing to a more noteworthy degree in bikes. This work can be reached out in a few ways. Inquiry in destiny work is identified with how the preparing is applied. Either the coping with could be applied to all arrangements disconnected once they have been accrued or a worldly window could be carried out earlier than playing out the department/grouping undertakings, which would likewise happen disconnected in a confined time location. This difficulty is specifically pivotal in genuine execution of the proposed approach. Plus, the received consequences are accomplished making use of the crude three-D accelerometers/particular speeds statistics. They may be improved by way of utilising highlight extraction/desire or facts mixture step. As destiny paintings, it may likewise be pertinent to utilize the contention among a version watching for the development of a framework nation and perceptions given by sensors introduced inside the PTW as a marker of the basic event happening. Utilizing strife inside the gadget of conviction paintings speculation licenses us to consider the sensor's imprecision and vulnerabilities.

\section{FUTURE WORK}

As a future augmentation of this work, the wellbeing framework can be incorporated with cameras for security purposes. The framework can likewise be coordinated with a calculation to discover the closest individual or emergency clinic to the spot of the mishap which will lessen the time wastage in giving clinical thoughtfulness regarding the harmed individual.

Published By:

Blue Eyes Intelligence Engineering

\& Sciences Publication

(C) Copyright: All rights reserved.

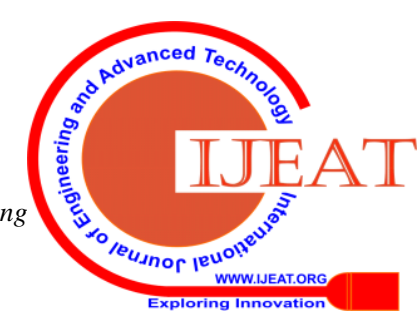




\section{REFERENCES}

1. Mohd Khairul Afiq Mohd Rasli, Nina Korlina Madzhi, Juliana Johari, "Smart helmet with sensors for twist of fate prevention ", in 2013 International Conference on Electrical,

2. Electronics and System Engineering (ICEESE), pp. 21-26

3. Nandu R \& Singh K, "Smart Helmet for Two Wheelers" Adv Automob Eng three:one hundred ten. Oct 2014,

4. doi:10.4172/2167-7670.1000110

5. Dr. G. N. Kodandaramaiah, Dr. Prabhakar V. Hunagund \& Mr. Santosh B Panjagal, "Smart

6. Helmet: The Next Generation Solar Gadget" IJMETMR, Vol no:2(2015), July 2015, ISSN No:2348-4845

7. ONISR. Observatoire National Inter ministeriel de l. A. SécuriteRoutière. Accessed: 2013. [Online]. Available: http://www.Securiteroutiere. Gouv.Fr/la-securite-routiere/l-observatoire-countrywideinterministeriel-de-lasecurite-routiere

8. SHRP2. (2015). Strategic Highway Research Program (SHRP2). Accessed: $2015 . \quad$ [Online]. Http://www.Trb.Org/StrategicHighwayResearch Program2SHRP2/Public/Blank2.Aspx

9. S. B. McLaughlin, Z. R. Doerzaph, and B. R. Cannon, "Pilot examine of instrumentation to collect behavioral records to perceive on-street rider behavior," Nat. Highway Traffic Saf. Admin., Washington, DC, USA, Tech. Rep. HS-811 442, 2011.

\section{AUTHORS’ PROFILE}

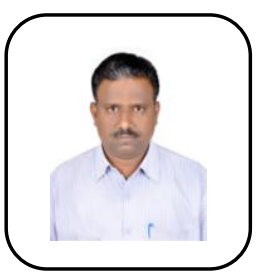

Kalyanaraman B, is an Assistant Professor in Department of Information Technology, SRMIST,Chennai,Tamil Nadu, India and has a teaching experience of 12 years

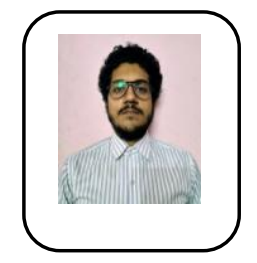

Shivam Choudhary, is currently pursuing bachelors of technology in information technology from SRM IST,Chennai, Tamil Nadu,India

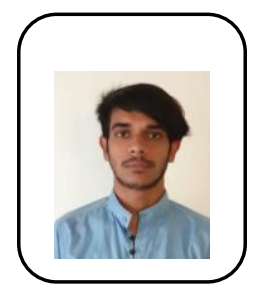

Malkeet Singh, is currently pursuing bachelors of technology in information technology from SRM IST,Chennai,Tamil Nadu,India

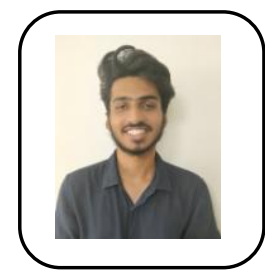

Kumar Divyank, is currently pursuing bachelors of technology in information technology from SRM IST,Chennai,Tamil Nadu,India 